\title{
Röntgenstrahlen - eine Alternative zu Medikamenten?
}

— Seit Jahrzehnten wird eine niedrig dosierte Strahlentherapie klinisch erfolgreich zur Behandlung entzündlicher und entzündlichdegenerativer Erkrankungen eingesetzt. Während die klinischen Behandlungskonzepte überwiegend auf frühen empirischen Studien beruhen, gelang es in den letzten Jahren zunehmend, durch Laboruntersuchungen und tierexperimentelle Studien zelluläre und

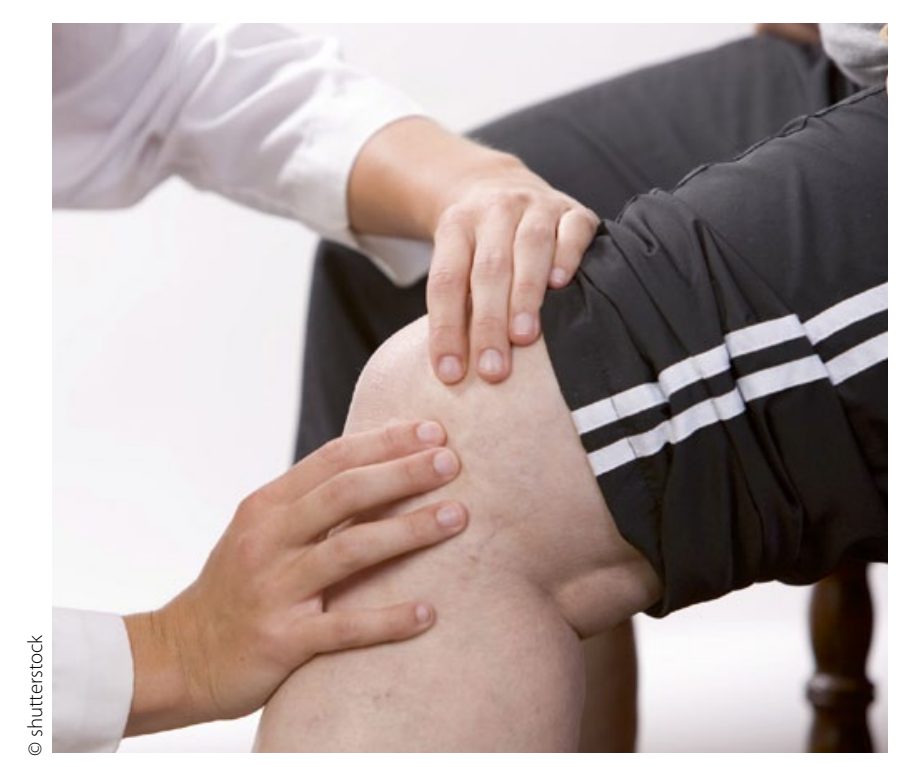

Wenn das Knie schmerzt: Niedrig dosierte Röntgenstrahlen als Alternative zu Medikamenten in Betracht ziehen. molekulare Mechanismen der Wirksamkeit zu identifizieren, die eine Rationale für den vermehrten klinischen Einsatz darstellen. Der zweite Schwerpunktbeitrag befasst sich daher eingehend mit dem aktuellen Stand der Wirkmechanismen.

Die Linderung der Symptomatik schmerzhafter entzündlicher oder degenerativer Prozesse an Gelenken und Weichteilen mit Hilfe von Röntgenstrahlen wurde in größeren Serien schon vor 80-90 Jahren berichtet. In den folgenden Jahrzehnten wurde die Strahlentherapie schmerzhafter, degenerativer und entzündlicher Erkrankungen in den deutschsprachigen Ländern weitgehend akzeptiert.

Der klinische Beitrag gibt einen Überblick über die in den letzten fünf Jahren gewonnen neuen Erkenntnisse und Veröffentlichungen, die zuletzt auf einem deutschsprachigen Symposium im April 2012 zusammengefasst wurden. Dabei wird ein aktueller Überblick über die häufigen Indikationen gegeben, die in die neu verabschiedete S2-Leitlinie "Strahlentherapie nicht maligner Erkrankungen" eingegangen sind.

Die in diesem MMW-Schwerpunkt dargestellten biologischen und klinischen Grundlagen zeigen, dass die niedrig dosierte Strahlentherapie eine effektive und gut verträgliche Behandlungsalternative bei schmerzhaften degenerativen und entzündlichen Veränderungen an Gelenken und Weichteilen ist, die zunehmend an Bedeutung gewinnt. 